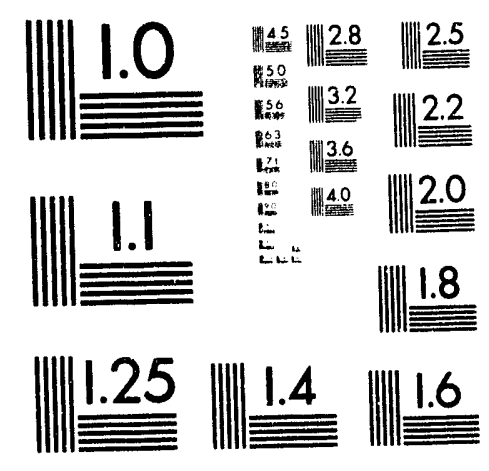



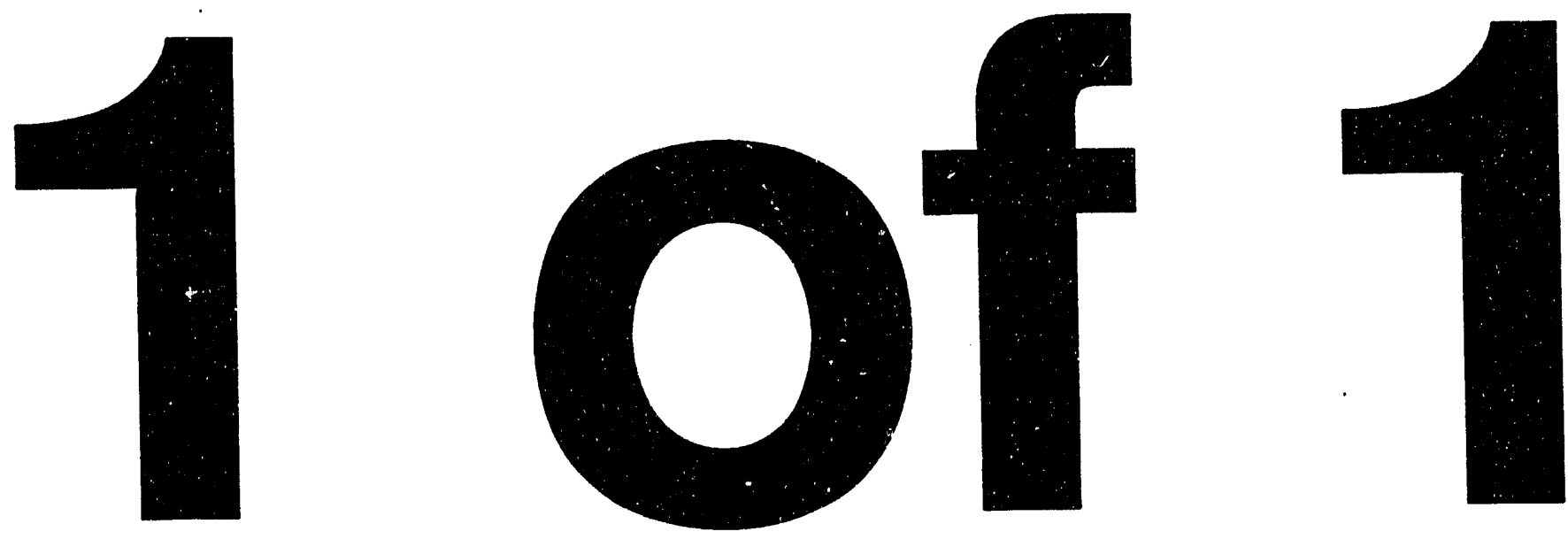
LBL-33312

$\mathrm{UC}-414$

\title{
Measurements of Higher-Order Mode Damping in the PEP-II Low-Power Test Cavity
}

\author{
R. A. Rimmer and D. A. Goldberg \\ Accelerator and Fusion Research Division \\ Lawrence Berkeley Laboratory \\ University of California \\ Berkeley, California 94720
}

May 1993

This work was supported by the Director, Office of Energy Research, Office of High Nuclear Physics, High Energy Physics Division, of the U.S. Department of Energy under Contract No. DE-AC03-76SF00098. 


\title{
Measurements of Higher-Order Mode Damping in the PEP-II Low-Power Test Cavity
}

\author{
R. A. Rimmer and D. A. Goldberg* \\ Lawrence Berkeley Laboratory \\ 1 Cyclotron Road, Berkeley, CA 94720, USA
}

\section{Abstract}

The paper describes the results of measurements of the HigherOrder Mode (HOM) spectrum of the low-power test model of the PEP-II RF cavity and the reduction in the Q's of the modes achieved by the addition of dedicated damping waveguides. All the longitudinal (monopole) and deflecting (dipole) modes below the beam pipe cut-off are identified by comparing their measured frequencies and field distributions with calculations using the URMEL code [1]. Field configurations were determined using a perturbation method with an automated bead positioning system [2]. The loaded Q's agree well with the calculated values reported previously, and the strongest HOMs are damped by more than three orders of magnitude. This is sufficient to reduce the coupled-bunch growth rates to within the capability of a reasonable feedback system. A highpower test cavity will now be built to validate the thermal design at the $150 \mathrm{~kW}$ nominal operating level, as described elsewhere at this conference [3].

\section{INTRODUCTION}

Impedances of higher order modes in the RF cavities can drive coupled-bunch instabilities in high-current storage rings. For the PEP-II $B$ factory [4] we have taken the approach that the HOMs must be damped to a point where a practical broadband feedback system can control the coupled-bunch growth rates. At the same time it is important to maximize the fundamental-mode impedance of the cavities to make most efficient use of RF power and to minimize the number of cavities and RF stations. The R\&D effort, shared among LBL, SLAC and LLNL, has produced a normal-conducting cavity design $[5,6]$ which uses a trio of dedicated damping waveguides to reduce the HOM impedances. The target was to reduce the longitudinal impedances to the order of a few $\mathrm{k} \Omega$, and the transverse impedances to a few hundred $\mathrm{k} \Omega / \mathrm{m}$ (comparable with the effect of the resistive-wall). The single-bunch parameters are typical of existing machines and not in the regime where microwave instabilities would be expected.

As a result of the ongoing $R \& D$ program some of the lattice and RF parameters have changed compared with those given in references 4,5 and 6 . All of the changes make the $R F$ design more conservative. Table 1 shows the current PEP-II RF system parameters.

*This work was supported by the Director, Office of Energy Research, Office of High Energy and Nuclear Physics, High Energy Physics Division of the U.S. Department of Energy, under Contracts DE-AC03-76SF00098 and DE-AC03-76SF00515.
Table 1

PEP-II RF system parameters (including the effect of the 5\% gap in the beam)

\begin{tabular}{|l|c|c|}
\hline PARAMETER & HER & LER \\
\hline \hline RF frequency (MHz) & 476 & 476 \\
Beam current (A) & 1.03 & 2.25 \\
Number of bunches & 1658 & $\geq 1658$ \\
Number of cavities & 20 & 10 \\
Shunt Impedance Rs $(\mathrm{M} \Omega)$ a & 3.5 & 3.5 \\
Gap Voltage (MV) & 0.93 & 0.60 \\
Accelerating gradient (MV/m) & 4.1 & 2.7 \\
Wall loss/cavity (kW) & 122 & 51 \\
Coupling factor without beam ( $\beta)$ & 7.5 & 7.5 \\
Unloaded Q of cavity & $\geq 30000$ & $\geq 30000$ \\
\hline
\end{tabular}

a $R_{s}=V^{2} / 2 P$

b with ports, at $40^{\circ} \mathrm{C}$

\section{LOW-POWER TEST CAVITY}

A low-power test cavity (LPTC) has been fabricated, see figure 1, which was designed to show the loading of the HOM Q's and, therefore, the impedance reduction that could be obtained with the waveguide damping scheme.

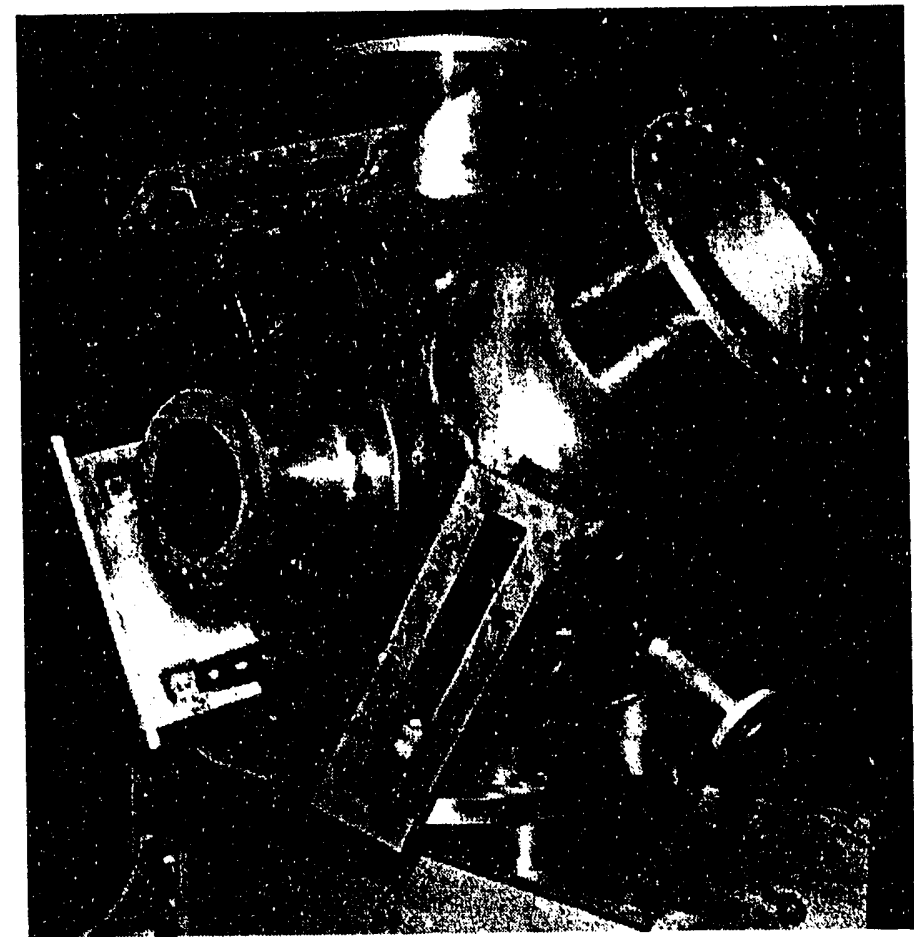

Figure 1. Low-power test cavity. The rectanguidr ports are for the HOM damping waveguides, the two large circular ports are alternative locations for a loop coupler. 
To save cost, the quality of construction, though good, was not intended to show the ultimate $\mathrm{Q}$ of the fundamental mode that could be expected in the final cavities. Low-power mock-ups of the waveguide loads were made using ferrite powder in epoxy, cast into long tapered wedges. The load material was placed at the edges of the waveguides at least 36 inches from the cavity, as would be the case in a high-power design, to avoid coupling to the evanescent fundamental mode.

\section{MEASUREMENTS}

The cavity was excited with small electric-field antennas inserted into the beam pipes and a Hewlett-Packard $8510 \mathrm{C}$ network analyzer was used to measure the transmission response $\left(S_{21}\right)$ between them. With all the apertures plugged or sealed, approximately flush with the cavity surface, the undamped modes of the cavity were seen as distinct peaks. The resonant frequencies were very close to those predicted by URMEL allowing easy identification of the modes.

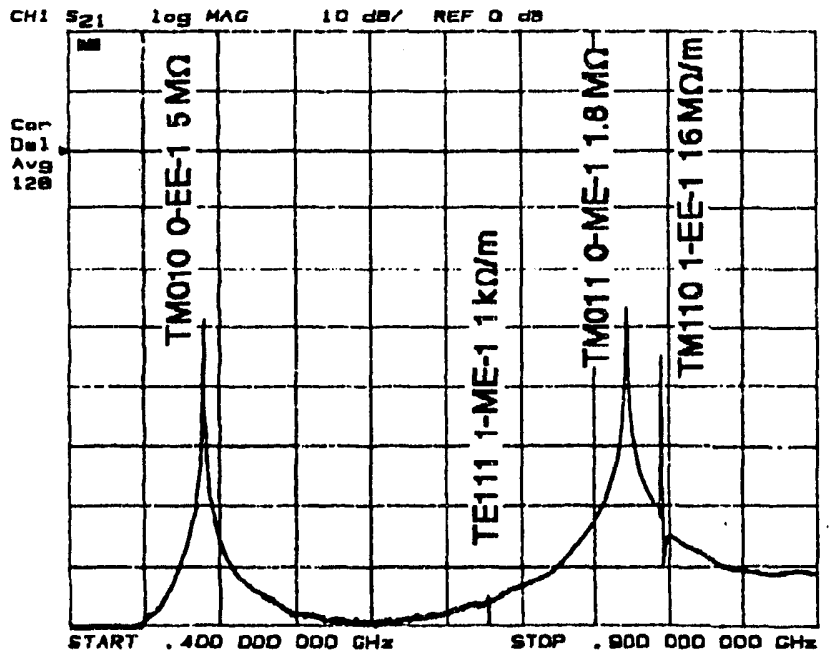

Figure 2. Fundamental mode and first three higher order modes in the LPTC without damping.

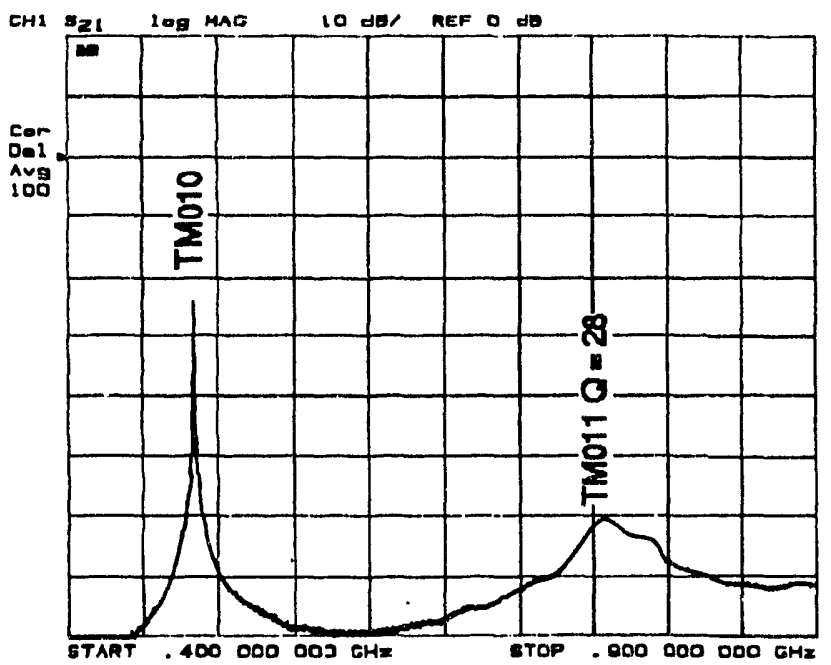

Figure 3. Fundamental mode and first higher order modes in the LPTC with three damping waveguides.
Figure 2 shows the spectrum of modes from below the fundamental (TM010) mode up to $900 \mathrm{MHz}$ without damping. The lowest TE mode (TE111-like), is visible, as are the TM011-like longitudinal mode (the strongest monopole HOM), at about $770 \mathrm{MHz}$, and the TM110-like deflecting (dipole) mode, at about $795 \mathrm{MHz}$. Figure 3 shows the same frequency span with the three damping waveguides attached. The fundamental mode is still strong while the HOMs are substantially damped. By changing the azimuthal position of the probes around the edges of the beam-pipes it is possible to enhance or reject the dipole modes relative to the monopole modes, and thereby measure the loaded Q' $s$ of overlapping monopole and dipole modes. It is also possible to discriminate between the two orientations of the dipole modes when there is a strong perturbation to the cavity such as the addition of the loop coupler.

Figure 4 shows more of the HOMs, between $900 \mathrm{MHz}$ and $1.4 \mathrm{GHz}$, without any damping. The monopole and dipole modes are labeled. Figure 5 shows the same span with the

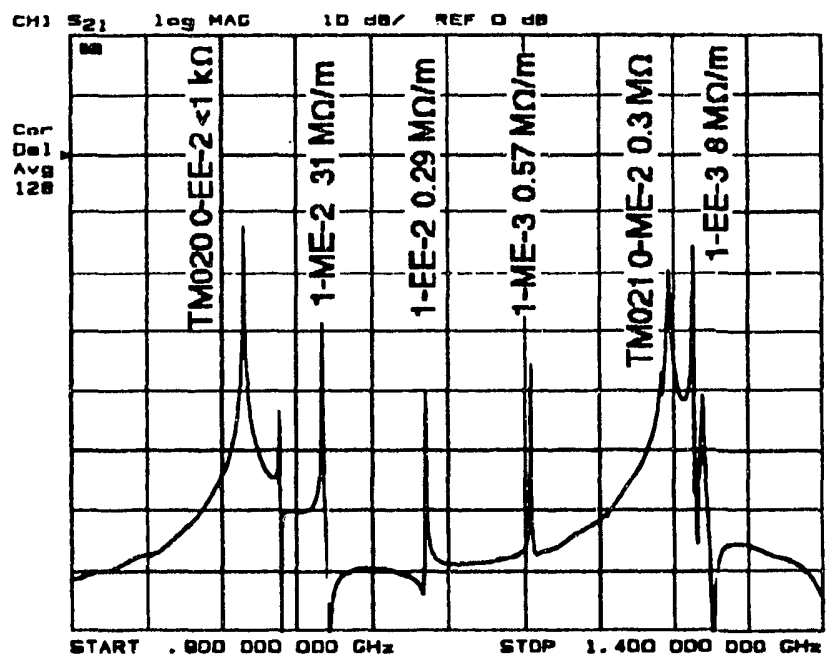

Figure 4. Higher order modes in the LPTC without damping, $0.9-1.4 \mathrm{GHz}$.

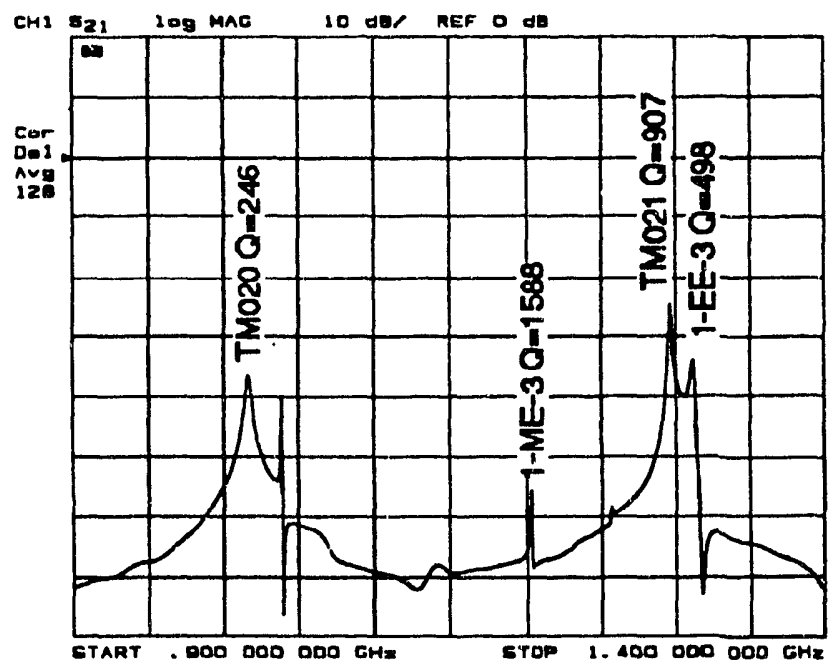

Figure 5. Higher order modes in the LPTC with three damping waveguides, $0.9-1.4 \mathrm{GHz}$. 
three damping waveguides. Still visible are the TM020-like monopole mode at about $1016 \mathrm{MHz}$, the TM021-like mode at about $1296 \mathrm{MHz}$ and the dipole mode at $1313 \mathrm{MHz}$. The residual impedances of these modes are within the capabilities of the feedback system. The results are similar for all the higher frequency HOMs up to the beam-pipe cut off. The residual impedances are shown in table 2 for the longitudinal modes and table 3 for the deflecting modes. A more detailed account of the results is available [7].

Table 2

Calculated and measured properties of longitudinal modes

\begin{tabular}{|c|c|c|c|c|c|c|}
\hline $\begin{array}{l}\text { Mode } \\
\text { type }\end{array}$ & $\begin{array}{c}\text { URMEL } \\
\text { name }\end{array}$ & $\begin{array}{c}\text { Calc Fo } \\
\text { (MHz) }\end{array}$ & $\begin{array}{l}\mathrm{R} / \mathrm{Q} \\
(\Omega) \\
\end{array}$ & $\begin{array}{r}\text { Meas FL } \\
(\mathrm{MHz})\end{array}$ & $\begin{array}{c}\text { Meas } \\
\mathrm{Q}\end{array}$ & $\begin{array}{r}R / L \\
(\mathrm{k} \Omega) \\
\end{array}$ \\
\hline TM010 & 0-EE-1 & 489.6 & 108.8 & 484 & *31926 & 3472 \\
\hline TM011 & $0-M E-1$ & 769.8 & 44.97 & 758 & 28 & 1.26 \\
\hline \multirow[t]{2}{*}{ TM020 } & 0-EE-2 & 1015.4 & 0.006 & 1016 & 246 & 0.001 \\
\hline & 0 -EE-3 & 1291.0 & 7.68 & no & longer & visible \\
\hline \multirow[t]{8}{*}{ TM021 } & 0-ME-2 & 1295.6 & 6.57 & 1296 & 907 & 5.96 \\
\hline & $0-E E-4$ & 1585.5 & 5.06 & 1588 & 178 & 0.90 \\
\hline & $0-\mathrm{ME}-3$ & 1711.6 & 4.75 & no & longer & visible \\
\hline & $0-E E-5$ & 1821.9 & 0.06 & 1821 & 295 & 0.018 \\
\hline & $0-\mathrm{ME}-4$ & 1891.0 & 1.68 & no & longer & visible \\
\hline & $0-\mathrm{EE}-6$ & 2103.4 & 3.52 & 2109 & 233 & 0.82 \\
\hline & $0-\mathrm{ME}-5$ & 2161.8 & 0.02 & 2168 & 201 & 0.004 \\
\hline & $0-\mathrm{EE}-7$ & 2252.2 & 1.21 & 2253 & 500 & 0.61 \\
\hline
\end{tabular}

*note that model does not test ultimate fundamental-mode $\mathrm{Q}$

Table 3

Calculated and measured properties of transverse modes

\begin{tabular}{|c|c|c|c|c|c|c|}
\hline $\begin{array}{l}\text { Mode } \\
\text { type }\end{array}$ & $\begin{array}{c}\text { URMEL } \\
\text { name }\end{array}$ & $\begin{array}{c}\text { Calc Fo } \\
(\mathrm{MHz})\end{array}$ & $\begin{array}{c}\mathrm{R} / \mathrm{Q}(\mathbf{k r}) \\
(\Omega)\end{array}$ & $\begin{array}{c}\text { Meas FL } \\
(\mathrm{MHz})\end{array}$ & $\begin{array}{c}\text { Meas } \\
Q_{L}\end{array}$ & $\begin{array}{l}\mathrm{R} \Perp \mathrm{L}^{*} \\
(\mathrm{k} \Omega / \mathrm{m})\end{array}$ \\
\hline $\begin{array}{l}\text { TE111 } \\
\text { TM110 }\end{array}$ & $\begin{array}{l}\text { 1-ME-1 } \\
\text { 1-EE-1 } \\
\text { 1-ME-2 } \\
\text { 1-EE-2 } \\
\text { 1-ME-3 } \\
\text { 1-EE-3 } \\
\text { 1-ME-4 } \\
\text { 1-EE-4 } \\
\text { 1-EE-5 } \\
\text { 1-EE-6 } \\
\text { 1-ME-5 } \\
\text { 1-ME-6 }\end{array}$ & \begin{tabular}{|r|}
679.6 \\
795.5 \\
1064.8 \\
1133.2 \\
1208.2 \\
1313.2 \\
1429.0 \\
1541.0 \\
1586.2 \\
1674.2 \\
1704.4 \\
1761.9
\end{tabular} & $\begin{array}{r}0.002 \\
15.263 \\
27.590 \\
0.243 \\
0.258 \\
5.861 \\
2.873 \\
0.850 \\
2.045 \\
5.140 \\
0.096 \\
0.104\end{array}$ & $\begin{array}{r}\text { no } \\
779 \\
\text { no } \\
1141 \\
1203 \\
1311 \\
1435 \\
1554 \\
1588 \\
1674 \\
1704 \\
1757\end{array}$ & $\begin{array}{r}\text { longer } \\
122 \\
\text { longer } \\
112 \\
1588 \\
498 \\
3955 \\
59 \\
178 \\
2134 \\
444 \\
7129\end{array}$ & $\begin{array}{c}\text { visible } \\
1.86 \\
\text { visible } \\
0.65 \\
10.3 \\
80.1 \\
341 \\
1.62 \\
12.1 \\
385 \\
1.52 \\
27.3\end{array}$ \\
\hline
\end{tabular}

$* \mathrm{R}_{\perp L}=\mathrm{R} / \mathrm{Q} /\left(\mathrm{kr}^{2}\right) \times \mathrm{QL}$, where $\mathrm{r}$ in the beam pipe radius

\section{MACHINE SIMULATIONS}

A simulation code has been used to study the growth rates of the longitudinal coupled-bunch instabilities that would be driven in PEP-II by the cavity impedances. The code includes a model of the beam dynamics, all of the residual HOM driving impedances from the cavities and a model of the proposed broad-band feedback system. Running the simulation for the longitudinal modes shows that the beam remains stable and that the large single-bunch transients that occur during injection are quickly damped down.

A transverse simulation program is being developed which is expected to show similar results for transverse beam motion since the deflecting impedances measured in the test cavity are reduced to the order of the resistive wall impedance.

\section{CONTINUING R\&D}

Studies of the low-power test model are continuing, in particular to evaluate the effects of the loop coupler which may provide additional damping for some of the HOMs. The automated bead-puller is being used to measure the field profiles of the modes and hence the R/Q's, and to study the perturbations introduced by the various ports. The PEP-II R\&D effort is now also focusing on building a high-power test model to prove that such a design can be conditioned and powered with up to $150 \mathrm{~kW}$ wall dissipation. High-power versions of the HOM loads are also being developed.

\section{CONCLUSIONS}

The low-power test model has shown that it is possible to achieve the HOM impedance reduction required for PEP-II with the broad-band waveguide damping scheme. The worst HOMs have been damped by more than three orders of magnitude and the loaded Q's agree well with the calculated values reported previously. The detailed design of a high-power test cavity is well under way.

\section{ACKNOWLEDGMENTS}

Although all the measurements were performed in the Lambertson Beam Electrodynamics Laboratory of the Center for Beam Physics at LBL, the design of the cavity has been very much a collaborative effort with the other participants in the PEP-II project. Thanks are due John Byrd at LBL for running the machine simulations.

\section{REFERENCES}

[1] "Reports at the 1986 Stanford Linac Conference., Stanford, USA, June 2-6 1986," DESY M-86-07, June 86

[2] D. A. Goldberg, R. A. Rimmer, "Automated BeadPositioning System for Measuring Impedances of RF Cavity Modes", this conierence.

[3] H. Schwarz et. al., "Design of a High-Power Test Model of the PEP-II RF Cavity", this conference.

[4] "An Asymmetric B-Factory based on PEP," Conceptual Design Report, LBL PUB-5303, SLAC 372

[5] R. Rimmer et. al., "An RF Cavity for the B-Factory", Proc. PAC, San Francisco, May 6th-9th, 2991, pp819-21.

[6] R. Rimmer et. al., "Higher Order Mode Damping Studies on the PEP-II B-Factory RF Cavity", Proc. 1992 Europ. Part. Accel. Conf., Berlin, Germany, March 24-28, 1992, pp 1289-1291.

[7] R. A. Rimmer, "RF Cavity Development for the PEP-II B Factory", Proc. Int. Workshop on B-Factories, BFWS92, KEK, Japan, Nov. 17-20, 1992, (PEP-II ME note 2-93, LBL-33360, ESG-242). 

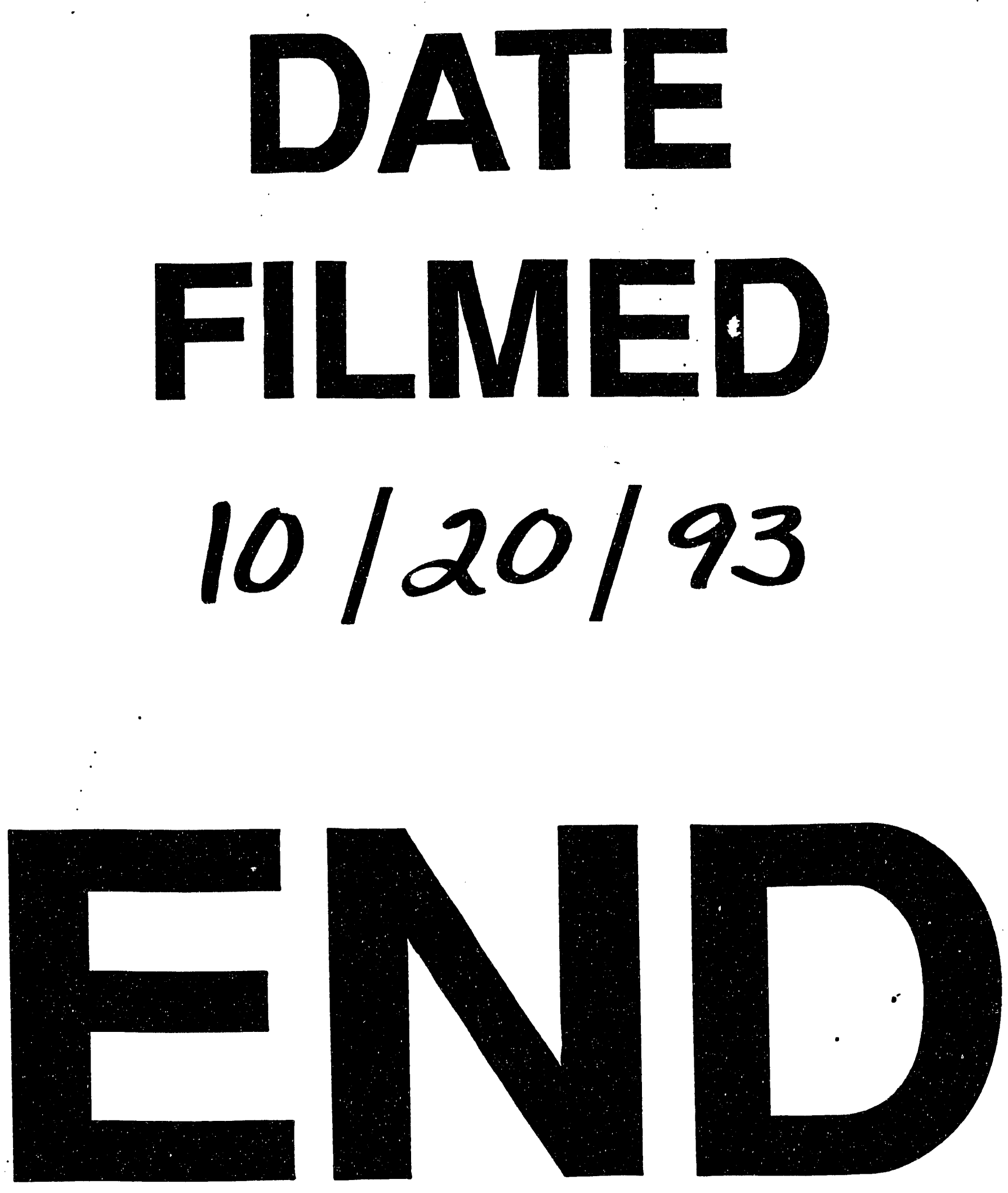
